\title{
Embedding relatively hyperbolic groups in products of trees
}

\author{
JOHN M MACKAY \\ AlESSANDRO Sisto
}

\begin{abstract}
We show that a relatively hyperbolic group quasi-isometrically embeds in a product of finitely many trees if the peripheral subgroups do, and we provide an estimate on the minimal number of trees needed. Applying our result to the case of 3manifolds, we show that fundamental groups of closed 3-manifolds have linearly controlled asymptotic dimension at most 8 . To complement this result, we observe that fundamental groups of Haken 3-manifolds with non-empty boundary have asymptotic dimension 2 .
\end{abstract}

20F65, 20F69

\section{Introduction}

If a group admits a quasi-isometric embedding into the product of finitely many trees then one can say a lot about the large scale geometry of the group. For example, this bounds the (linearly controlled) asymptotic dimension of the group. It has implications for the Hilbert compression of the group, and also the topological dimension of any asymptotic cone. Such embeddings have recently been constructed for hyperbolic groups; see Buyalo [12], and Buyalo, Dranishnikov and Schroeder [14].

In this paper we show that if every peripheral group of a relatively hyperbolic group embeds into the product of finitely many trees, then the entire group does. Moreover, we give explicit bounds for the number of trees required.

We begin by recalling what is known for hyperbolic groups. In this case, the situation has been completely understood by work of Buyalo and Lebedeva [15, Theorems 1.5, 1.6]. (For further refinements, see [14].)

Theorem 1.1 Let $G$ be a Gromov hyperbolic group. Then $G$ admits a quasi-isometric embedding into the product of $n+1$ metric trees, where $n=\operatorname{dim} \partial_{\infty} G$ is the topological dimension of the boundary. Moreover, $G$ does not embed into any product of $n$ metric trees. 
Our main result is the following. (For the definition of a relatively hyperbolic group, see Section 2.)

Theorem 1.2 Suppose the group $G$ is hyperbolic relative to subgroups $H_{1}, \ldots, H_{n}$. If each $H_{i}$ quasi-isometrically embeds into a product of $m$ metric trees, then $\operatorname{asdim}(G)<$ $\infty$ and $G$ quasi-isometrically embeds into a product of $M$ metric trees, where

$$
M=\max \{\operatorname{asdim}(G), m+1\}+m+1<\infty .
$$

Conversely, if $G$ quasi-isometrically embeds into a product of $N$ metric trees, then each peripheral group does also.

The assertion $\operatorname{asdim}(G)<\infty$ follows from Osin [39].

Both these theorems use results that study the relationship between the asymptotic dimension of a hyperbolic space and the linearly controlled metric dimension of its boundary. We now proceed to define these and related concepts. (See Buyalo and Schroeder [17] for more discussion.)

Suppose $\mathcal{U}$ is a family of subsets of a metric space $X$. We say $\mathcal{U}$ is $D$-bounded if the diameter of every $U \in \mathcal{U}$ is at most $D$. The $r$-multiplicity of $\mathcal{U}$ is the infimal integer $n$ so that every subset of $X$ with diameter less than or equal to $r$ meets at most $n$ subsets of $\mathcal{U}$.

The asymptotic dimension of $X$, denoted by $\operatorname{asdim}(X)$, is the smallest $n \in \mathbb{N} \cup\{\infty\}$ so that for all $r>0$, there exists $D(r)<\infty$ and a $D(r)$-bounded cover $\mathcal{U}$ of $X$ with $r$-multiplicity at most $n+1$; see Gromov [28, 1.E]. The linearly controlled asymptotic dimension of $X$, denoted by $\ell$-asdim $(X)$, is the smallest $n$ so that there exists $L<\infty$ with the property that for all sufficiently large $r<\infty$, there exists an $L r$-bounded cover $\mathcal{U}$ of $X$ with $r$-multiplicity at most $n+1$ (Lang and Schlichenmaier [35], and Brodskiy, Dydak, Higes and Mitra [8]). (This is sometimes called "asymptotic Assouad-Nagata dimension" in the literature.)

The following definition simplifies our discussion of how metric spaces embed in trees. (For the related notion of " $t$-rank", see Buyalo and Schroeder [17, page 176].)

Definition 1.3 Given a metric space $X$, let eco-dim $(X)$ be the smallest $n \in \mathbb{N}$ so that $X$ quasi-isometrically embeds in the product of $n$ metric trees, and set eco-dim $(X)=\infty$ if no such embedding exists.

The inequalities $\operatorname{asdim}(X) \leq \ell-\operatorname{asdim}(X) \leq$ eco-dim $(X)$ hold for any metric space $X$. These three quantities are equal for hyperbolic groups, as shown in Buyalo and Lebedeva 
[15]. Both equalities can fail in general: there are groups $G$ with finite asymptotic dimension but infinite linearly controlled asymptotic dimension; see Nowak [38]. The discrete Heisenberg group $H$ has $\operatorname{asdim}(H)=\ell-\operatorname{asdim}(H)=3$, but eco-dim $(H)=\infty$ (see the discussion in Section 5).

As an aside, Lang and Schlichenmaier show that if $\ell$-asdim $(X, d)<\infty$, then for sufficiently small $\epsilon>0$, the snowflaked space $\left(X, d^{\epsilon}\right)$ has

$$
\operatorname{eco-dim}\left(X, d^{\epsilon}\right) \leq \ell-\operatorname{asdim}(X, d)+1
$$

\section{[35, Theorem 1.3].}

For hyperbolic groups, these asymptotic invariants are related to the local properties of the boundary. The linearly controlled metric dimension of $X$, denoted by $\ell-\operatorname{dim}(X)$, is the smallest $n$ so that there exists $L<\infty$ with the property that for all sufficiently small $r>0$, there exists an $L r$-bounded cover $\mathcal{U}$ of $X$ with $r$-multiplicity at most $n+1$ [12, Proposition 3.2]. (This is also referred to as "capacity dimension".) Buyalo shows the following embedding theorem.

Theorem 1.4 [13, Theorem 1.1] Suppose $X$ is a visual, Gromov hyperbolic metric space, and $\ell-\operatorname{dim}\left(\partial_{\infty} X\right)<\infty$. Then eco-dim $(X) \leq \ell-\operatorname{dim}\left(\partial_{\infty} X\right)+1$.

The following proposition gives a simple bound for the linearly controlled metric dimension of the boundary of $X$.

Proposition 3.6 Suppose $X$ is a Gromov hyperbolic geodesic metric space. Then $\ell-\operatorname{dim}\left(\partial_{\infty} X\right) \leq \operatorname{asdim}(X)$.

This proposition does not seem to be recorded in the literature, possibly because in the case when the space admits a cocompact isometric action the stronger equality $\operatorname{asdim}(X)=\ell-\operatorname{dim}\left(\partial_{\infty} X\right)+1$ holds [15]. Even without such an action, Buyalo shows the inequality $\operatorname{asdim}(X) \leq \ell-\operatorname{dim}\left(\partial_{\infty} X\right)+1[12]$.

In the case of a relatively hyperbolic group $\left(G,\left\{H_{i}\right\}\right)$, a natural choice for $X$ is $X(G)$, the Bowditch space associated to $\left(G,\left\{H_{i}\right\}\right)$; see Definition 2.3. This is a visual, Gromov hyperbolic, geodesic metric space. We bound the asymptotic dimension of $X(G)$ in terms of the asymptotic dimension of $G$ and the linearly controlled asymptotic dimension of the peripheral groups.

Proposition 3.4 Let $G$ be hyperbolic relative to $H_{1}, \ldots, H_{n}$, and let $m$ be the maximum of $\ell-\operatorname{asdim}\left(H_{i}\right)$ for $i=1, \ldots, n$. Then

$$
\max \{\operatorname{asdim}(G), m\} \leq \operatorname{asdim}(X(G)) \leq \max \{\operatorname{asdim}(G), m+1\} .
$$


Osin had earlier shown that $\operatorname{asdim}(G)$ is finite if $\operatorname{asdim}\left(H_{i}\right)<\infty$ for each $i$ [39, Theorem 1.2].

The product of $n$ (unbounded) metric trees has linearly controlled asymptotic dimension $n$ (see, eg, Lang and Schlichenmaier [35]). Therefore, Theorem 1.4 and Propositions 3.6 and 3.4 combine to show the following.

Corollary 1.5 Suppose the group $G$ is hyperbolic relative to $H_{1}, \ldots, H_{n}$. Let $m=$ $\max \left\{\operatorname{eco-dim}\left(H_{i}\right)\right\}$. Then eco-dim $(X(G)) \leq \max \{\operatorname{asdim}(G), m+1\}+1$.

In order to prove Theorem 1.2, we use work of Bestvina, Bromberg and Fujiwara to combine the embeddings of the peripheral groups and of $X(G)$ into a single embedding of $G$ into a product of trees.

Theorem 4.1 Let $G$ be hyperbolic relative to $H_{1}, \ldots, H_{n}$, and suppose that each $H_{i}$ admits a quasi-isometric embedding into the product of $m$ trees. Then $G$ admits a quasiisometric embedding into the product of $m$ trees and either $X(G)$ or the coned-off graph $\widehat{G}$.

This theorem completes the proof of Theorem 1.2. Note that the converse statement is automatic, as peripheral groups of a relatively hyperbolic group are undistorted in the ambient group by Druţu and Sapir [24, Lemma 4.15].

Rather than using the Bowditch space $X(G)$, to improve the bounds in Theorem 1.2 one might hope to use instead the (hyperbolic) coned-off graph $\hat{G}$. However, the non-locally finite nature of $\widehat{G}$ leads to a non-compact boundary, and much of the machinery used above no longer applies.

The embeddings we consider are not and cannot be required to be equivariant, because there exist hyperbolic groups with property (T) (for example, any cocompact lattice in $S p(n, 1)$ ), which in particular cannot act interestingly on trees. We remark that the inverse problem of equivariantly embedding trees into hyperbolic spaces is treated in Burger, Iozzi and Monod [11].

Theorem 1.2 has the following immediate corollary.

Corollary 1.6 If the peripheral groups of a relatively hyperbolic group $G$ each quasiisometrically embed into the product of finitely many trees, then $\ell$-asdim $(G)<\infty$.

If the group $G$ satisfies $\ell$-asdim $(G)<\infty$ then it has Hilbert compression 1 by Gal [27, Theorem 1.1.1]. For more general estimates on the Hilbert compression of a relatively hyperbolic group, see Hume [31].

In Section 5 we apply our results to 3-manifold groups, and show, amongst other results, the following. 
Theorem 5.1 Let $G=\pi_{1}(M)$, where $M$ is a compact, orientable 3-manifold whose (possibly empty) boundary is a union of tori. Then eco-dim $(G)<\infty$ if and only if no manifold in the prime decomposition of $M$ has Nil geometry; in this case, $\operatorname{eco}-\operatorname{dim}(G) \leq 8$.

In any case, $\ell-\operatorname{asdim}(G) \leq 8$.

Finally, in Appendix A we prove a bound on the asymptotic dimension of HNN extensions, following work of Dranishnikov.

\subsection{Recent developments}

Since the appearance of the first version of this paper, Hume [32] has shown that the construction of Bestvina, Bromberg and Fujiwara that we use in Section 4 gives rise to spaces that are quasi-isometric to a tree-graded space. Tree-graded spaces are defined in Druţu and Sapir [24]; roughly speaking, they are tree-like arrangements of certain specified subspaces called pieces (for example, consider a Cayley graph of a free product). It is known by Brodskiy and Higes [10] that if $X$ is tree-graded and $\mathcal{P}$ is its collection of pieces then

$$
\ell-\operatorname{asdim}(X) \leq \max _{P \in \mathcal{P}}\{1, \ell-\operatorname{asdim}(P)\}
$$

when, say, there are finitely many $(L, C)$-quasi-isometry types of pieces, for some given $L, C$.

As noticed in [32], the arguments in Section 4 (just considering $\pi_{Y}$ instead of $f_{i, Y} \circ \pi_{Y}$ in Section 4.2), combined with [32], give the following.

Theorem 1.7 (cf. Theorem 4.1) Let $G$ be hyperbolic relative to $H_{1}, \ldots, H_{n}$. Then $G$ quasi-isometrically embeds in the product of a space $\mathcal{T}$ and either the coned-off graph $\hat{G}$ or the Bowditch space $X(G)$, where $\mathcal{T}$ is tree-graded and each piece is uniformly quasi-isometric to some $H_{i}$.

In particular, once again as noticed in [32], using the aforementioned result from [10] one can get the following.

Corollary 1.8 A relatively hyperbolic group has finite $\ell$-asdim if and only if its peripheral subgroups do. 


\section{Acknowledgements}

We thank Alexander Dranishnikov, David Hume, Urs Lang, Enrico Le Donne and the referee for helpful comments, and Marc Lackenby for providing a fix for the proof of Proposition 5.2. The authors were supported by EPSRC grant "Geometric and analytic aspects of infinite groups".

\section{Relatively hyperbolic groups}

In this section we define relatively hyperbolic groups and their (Bowditch) boundaries.

\subsection{Definitions}

There are many definitions of relatively hyperbolic groups. We will give one in terms of actions on a cusped space. First we define our model of a horoball.

Definition 2.1 Suppose $\Gamma$ is a connected graph with vertex set $V$ and edge set $E$, where every edge has length one. The horoball $\mathcal{H}(\Gamma)$ is defined to be the graph with vertex set $V \times \mathbb{N}$ and edges $((v, n),(v, n+1))$ of length 1 , for all $v \in V, n \in \mathbb{N}$, and edges $\left((v, n),\left(v^{\prime}, n\right)\right)$ of length $e^{-n}$, for all $\left(v, v^{\prime}\right) \in E$.

Note that $\mathcal{H}(\Gamma)$ is quasi-isometric to the metric space constructed from $\Gamma$ by gluing to each edge in $E$ a copy of the strip [0,1] $\times[1, \infty)$ in the upper half-plane model of $\mathbb{H}^{2}$, where the strips are attached to each other along $v \times[1, \infty)$.

As is well known, these horoballs are hyperbolic with boundary a single point. Moreover, it is easy to estimate distances in horoballs. We will write $A \approx B$ if the quantities $A$ and $B$ differ by some constant.

Lemma 2.2 Suppose $\Gamma$ and $\mathcal{H}(\Gamma)$ are defined as above. Let $d_{\Gamma}$ and $d_{\mathcal{H}}$ denote the corresponding path metrics. Then for each $(x, m),(y, n) \in \mathcal{H}(\Gamma)$, we have

$$
d_{\mathcal{H}}((x, m),(y, n)) \approx 2 \ln \left(d_{\Gamma}(x, y) e^{-\min \{m, n\}}+1\right)+|m-n| .
$$

Proof We may assume that $m \leq n$. We can suppose $d_{\Gamma}(x, y) \geq e^{m}$, as if not, $|m-n| \leq$ $d_{\mathcal{H}}((x, m),(y, n)) \leq 1+|m-n|$. In particular $\ln \left(d_{\Gamma}(x, y) / e^{m}+1\right) \approx \ln d_{\Gamma}(x, y)-m$. By construction of $\mathcal{H}(\Gamma)$, any geodesic $\gamma$ between $(x, m)$ and $(y, n)$ in $\mathcal{H}(\Gamma)$ must go from $(x, m)$ to $(x, t)$ changing only the second coordinate, then follow a geodesic $\gamma^{\prime} \subset \Gamma \times\{t\} \subset \mathcal{H}(\Gamma)$ to $(y, t)$, then back to $(y, n)$. Thus

$$
d_{\mathcal{H}}((x, m),(y, n)) \leq 2(t-m)+|n-m|+e^{-t} d_{\Gamma}(x, y),
$$


with equality for the best choice of $t$. It is readily seen that this value is attained for the least $t$ so that $l_{t}=e^{-t} d_{\Gamma}(x, y)$ satisfies $l_{t} / e+2 \geq l_{t}$, that is, $l_{t} \leq 2 e /(e-1)=\rho$. So the best choice of $t$ is $t=\left\lceil\ln \left(d_{\Gamma}(x, y) / \rho\right)\right\rceil$, and the right hand side of (2-1) is $2\left(\ln d_{\Gamma}(x, y)-m\right)-2 \ln \rho+|n-m|+\epsilon$, where $|\epsilon| \leq 2+\rho$.

Definition 2.3 Suppose $G$ is a finitely generated group, and $\left\{H_{i}\right\}_{i=1}^{n}$ a collection of finitely generated subgroups of $G$. Let $S$ be a finite generating set for $G$, so that $S \cap H_{i}$ generates $H_{i}$ for each $i=1, \ldots, n$.

Let $\Gamma(G, S)$ be the Cayley graph of $G$ with respect to $S$. Let $X(G)=X\left(G,\left\{H_{i}\right\}, S\right)$ be the space resulting from gluing to $\Gamma(G, S)$ a copy of $\mathcal{H}\left(\Gamma\left(H_{i}, S \cap H_{i}\right)\right)$ to each coset $g H_{i}$ of $H_{i}$, for each $i=1, \ldots, n$. We call $X(G)$ the Bowditch space associated to $\left(G,\left\{H_{i}\right\}, S\right)$.

We say that $\left(G,\left\{H_{i}\right\}\right)$ is relatively hyperbolic if $X(G)$ is Gromov hyperbolic, and call the members of $\left\{H_{i}\right\}$ peripheral subgroups.

This is equivalent to the other usual definitions of (strong) relative hyperbolicity; see Bowditch [7] and Groves and Manning [29, Theorem 3.25].

\subsection{Visual metric}

Let $X$ be a geodesic, Gromov hyperbolic space (not necessarily proper), with fixed base point $0 \in X$. Suppose all geodesic triangles are $\delta$-slim. One equivalent definition of the boundary $\partial_{\infty} X$ is as the set of equivalence classes of $(1,20 \delta)$-quasigeodesic rays $\gamma:[0, \infty) \rightarrow X$, with $\gamma(0)=0$, where two rays are equivalent if they are at finite Hausdorff distance from each other. Let $(x \mid y)=(x \mid y)_{0}$ denote the Gromov product on $\partial_{\infty} X$ with respect to 0 . Up to an additive error, $(x \mid y)$ equals the distance from 0 to some (any) $(1,20 \delta)$-quasigeodesic line from $x$ to $y$; see Kapovich and Benakli [34, Remark 2.16].

A metric $\rho$ on $\partial_{\infty} X$ is a visual metric if there exists $C_{0}, \epsilon>0$ so that

$$
\frac{1}{C_{0}} e^{-\epsilon(x \mid y)} \leq \rho(x, y) \leq C_{0} e^{-\epsilon(x \mid y)}
$$

for all $x, y \in \partial_{\infty} X$. Boundaries of Bowditch spaces will always be endowed with a visual metric. The linearly controlled metric dimension of such boundary is independent of the choice of visual metric; see Buyalo [13].

For more results on the geometric properties of $\partial_{\infty} X(G)$, see Mackay and Sisto [37]. 


\subsection{Distance formula}

Let $G$ be a relatively hyperbolic group and let $\mathbb{Y}$ be the collection of all left cosets of peripheral subgroups. Fix a Cayley graph of $G$ with path metric $d$. For $Y \in \mathbb{Y}$, let $\pi_{Y}: G \rightarrow Y$ be a closest point projection map onto $Y$ with respect to $d$. Denote by $\widehat{G}$ the coned-off graph of $G$, that is to say the metric graph obtained from the Cayley graph of $G$ by adding an edge of length one connecting each pair of (distinct) vertices contained in the same left coset of peripheral subgroup. The path metric on $\widehat{G}$ is denoted by $d_{\widehat{G}}$. Let $\{\{x\}\}_{L}$ denote $x$ if $x>L$, and 0 otherwise. We write $A \approx_{\lambda, \mu} B$ if $A / \lambda-\mu \leq B \leq \lambda A+\mu$. The following is proven in Sisto [43].

Theorem 2.4 (Distance formula for relatively hyperbolic groups) There exists $L_{0}$ so that for each $L \geq L_{0}$ there exist $\lambda, \mu$ so that the following holds. If $x, y \in G$ then

$$
d(x, y) \approx_{\lambda, \mu} \sum_{Y \in \mathbb{Y}}\left\{\left\{d\left(\pi_{Y}(x), \pi_{Y}(y)\right)\right\}\right\}_{L}+d_{\widehat{G}}(x, y) .
$$

\section{Large scale and boundary dimension estimates}

In this section we bound the asymptotic dimension of the Bowditch space of a relatively hyperbolic group. We also bound the linearly controlled metric dimension of the boundary of a Gromov hyperbolic space by its asymptotic dimension.

Observe that at the cost of a slight relaxation in the value of $r$, a collection of subsets $\mathcal{U}$ has $r$-multiplicity at most $m$ if and only if we can write $\mathcal{U}=\bigcup_{i=1}^{m} \mathcal{U}_{i}$, where each $\mathcal{U}_{i}$ is $r$-disjoint: it is a collection of subsets pairwise separated by a distance of at least $r$. This follows from an application of Zorn's lemma. We will use this alternative characterization throughout this section.

\subsection{Asymptotic dimension of Bowditch spaces}

First we bound the asymptotic dimension of a horoball.

Proposition $3.1 \operatorname{asdim}(\mathcal{H}(\Gamma)) \leq \ell-\operatorname{asdim}(\Gamma)+1$.

Proof We will use the Hurewicz theorem for asymptotic dimension.

Theorem 3.2 [5, Theorem 1] Let $f: X \rightarrow Y$ be a Lipschitz map between geodesic metric spaces, and suppose that for each $R$ the family $\left\{f^{-1}\left(B_{R}(y)\right)\right\}_{y \in Y}$ has uniform asymptotic dimension $\leq m$. Then $\operatorname{asdim}(X) \leq \operatorname{asdim} Y+m$. 
Choose some vertex $x \in \Gamma$, and let $\gamma$ be the geodesic ray in $\mathcal{H}(\Gamma)$ obtained by concatenating the edges between $(x, i)$ and $(x, i+1)$ for all $i \in \mathbb{N}$. Let $f: \mathcal{H}(\Gamma) \rightarrow \gamma$ be the natural 1 -Lipschitz map given by $f(z, i)=(x, i)$. Fix some $R>0$. The preimages under $f$ of all balls of radius $R$ in $\gamma$ are quasi-isometric (with constants depending on $R$ only) to $\left(\Gamma, d_{n}\right)$ for some $n$, where $d_{n}(x, y)=2 \ln \left(d_{\Gamma}(x, y) e^{-n}+1\right)$, see Lemma 2.2. So, we only need to show that the uniform asymptotic dimension of $\left\{\left(\Gamma, d_{n}\right)\right\}$ is at most $m$. Fix any $R$ large enough. There exists $C=C(\Gamma)$ so that the following holds. Let $m=\ell$-asdim $(\Gamma)$. For each $n$ there exists a covering $\mathcal{U}(n)=\mathcal{U}_{1}(n) \cup \cdots \cup \mathcal{U}_{m+1}(n)$ of $\left(\Gamma, d_{\Gamma}\right)$ such that each $\mathcal{U}_{i}(n)$ is $e^{n+R}$-disjoint and $C e^{n+R}$-bounded. So, $\mathcal{U}_{i}(n)$ is $(2 R-M)$-disjoint and $(2 R+M)$-bounded with respect to $d_{n}$, where $M$ depends on $\Gamma$ only.

Proposition 3.1 has a weak converse.

Proposition $3.3 \quad \ell$-asdim $(\Gamma) \leq \operatorname{asdim}(\mathcal{H}(\Gamma))$.

Proof Set $m=\operatorname{asdim}(\mathcal{H}(\Gamma))$. There exists a covering $\mathcal{U}=\mathcal{U}_{1} \cup \cdots \cup \mathcal{U}_{m+1}$ of $\mathcal{H}(\Gamma)$ such that each $\mathcal{U}_{i}$ is $r$-disjoint and $R$-bounded, for some $r, R$ large enough. Up to increasing $R$ and decreasing $r$ we have that for each $n$ there exists a covering $\mathcal{U}_{1}(n) \cup \cdots \cup \mathcal{U}_{m+1}(n)$ of $\left(\Gamma, d_{n}\right)$ with the same properties, where $d_{n}(x, y)=$ $2 \ln \left(d_{\Gamma}(x, y) e^{-n}+1\right)$ as in the previous proof. So, each $\mathcal{U}_{i}(n)$ is $e^{n}\left(e^{r / 2}-1\right)$-disjoint and $e^{n}\left(e^{R / 2}-1\right)$-bounded in $\left(\Gamma, d_{\Gamma}\right)$. We are done as

$$
\frac{e^{n}\left(e^{R / 2}-1\right)}{e^{n}\left(e^{r / 2}-1\right)}
$$

is bounded independently of $n$.

Now we can bound the asymptotic dimension of the Bowditch space.

Proposition 3.4 Let $G$ be hyperbolic relative to $H_{1}, \ldots, H_{n}$, and let $m$ be the maximum of $\ell$-asdim $\left(H_{i}\right)$ for $i=1, \ldots, n$. Then

$$
\max \{\operatorname{asdim}(G), m\} \leq \operatorname{asdim}(X(G)) \leq \max \{\operatorname{asdim}(G), m+1\} .
$$

Proof The lower bound by $m$ follows from Proposition 3.3. The lower bound by $\operatorname{asdim}(G)$ follows as $X(G)$ contains $G$ with a proper metric.

For the upper bound, we will use the union theorem for asymptotic dimension. 
Theorem 3.5 (Bell and Dranishnikov [3, Theorem 1]) Let $Y$ be a geodesic metric space and suppose that $Y=\bigcup_{i \in \mathbb{N}} A_{i}$. Also, suppose that $\left\{A_{i}\right\}$ has uniform asymptotic dimension at most $n$ and for each $R$ there exists $Y_{R} \subseteq Y$ so that asdim $Y_{R} \leq n$ and $\left\{A_{i} \backslash Y_{R}\right\}$ is $R$-disjoint. Then $\operatorname{asdim} Y \leq n$.

We apply the theorem with $\left\{A_{i}\right\}$ the family of horoballs of $X(G)$ and for each $R$, take $Y_{R}$ to be a suitable neighborhood of an orbit of $G$. By Proposition 3.1, $\operatorname{asdim}\left(A_{i}\right) \leq$ $m+1$, and the constants are uniform as there are only finitely many different isometry types. Finally, $\operatorname{asdim}\left(Y_{R}\right)=\operatorname{asdim}(G)$ because the action of $G$ on $X(G)$ is proper.

\subsection{Linearly controlled metric dimension estimate}

Proposition 3.6 Suppose $X$ is a Gromov hyperbolic geodesic metric space. Then $\ell-\operatorname{dim}\left(\partial_{\infty} X\right) \leq \operatorname{asdim}(X)$.

Proof We fix the notation of Section 2.2 regarding visual metrics, and write $d$ for the metric on $X$.

Each $x \in \partial_{\infty} X$ is the limit of some $(1,20 \delta)$-quasigeodesic $\gamma$ [34, Remark 2.16]. Given $R>0$, define the projection $\pi_{R}: \partial_{\infty} X \rightarrow X$ by $\pi_{R}(x)=\gamma(R)$. This is well defined up to an error of $C_{1}=C_{1}(\delta)$. By considering a quasi-geodesic triangle between $a, b$ and 0 , observe that there exists $C_{2}=C_{2}\left(C_{0}, C_{1}, \delta\right)$ so that if $d\left(\pi_{R}(a), \pi_{R}(b)\right) \geq$ $s>2 C_{1}$, then $\rho(a, b) \geq\left(1 / C_{2}\right) e^{-\epsilon(R-s / 2)}$. Similarly, if $d\left(\pi_{R}(a), \pi_{R}(b)\right) \leq t$ then $\rho(a, b) \leq C_{2} e^{-\epsilon(R-t / 2)}$.

If $\operatorname{asdim}(X) \leq n$, then, given $s=3 C_{1}$, there exists $t<\infty$ and a cover $\mathcal{U}=\bigcup_{0}^{n} \mathcal{U}_{i}$ so that each $U \in \mathcal{U}$ has diameter at most $t$, and $\mathcal{U}_{i}$ is $s$-disjoint.

Suppose some small $r>0$ is given. Let $R=-\frac{1}{\epsilon} \ln r$. For $U \in \mathcal{U}_{i} \subset \mathcal{U}$, let $\widehat{U} \subset \partial_{\infty} X$ be the set of points $z$ so that there exists a $(1,20 \delta)$-quasigeodesic $\gamma$ from 0 to $z$ with $\gamma(R) \in U$. Let $\widehat{\mathcal{U}}_{i}=\bigcup_{U \in \mathcal{U}_{i}} \hat{U}$, and $\widehat{\mathcal{U}}=\bigcup_{i=1}^{n} \widehat{\mathcal{U}}_{i}$.

By the above estimates, $\hat{\mathcal{U}}_{i}$ is $\left(e^{\epsilon S / 2} r / C_{2}\right)$-disjoint, and $\hat{\mathcal{U}}$ is $C_{2} r e^{\epsilon t / 2}$-bounded. Since the ratio of these distances is bounded by $C_{2}^{2} e^{\epsilon(t-s) / 2}$, we have $\ell-\operatorname{dim}\left(\partial_{\infty} X\right) \leq n$.

\section{Embedding in a product of quasi-trees and the Bowditch space}

The aim of this section is to find an embedding of a given relatively hyperbolic group into a product of trees "stabilized" by the Bowditch space or the coned-off graph. 
Theorem 4.1 Let $G$ be hyperbolic relative to $H_{1}, \ldots, H_{n}$. Suppose there exists $k$ so that each $H_{i}$ admits quasi-isometric embedding into the product of $k$ trees. Then $G$ admits a quasi-isometric embedding into the product of $k$ trees and either $X(G)$ or the coned-off graph $\widehat{G}$.

We prove this theorem in Section 4.2.

\subsection{Quasi-trees of spaces}

To prove Theorem 4.1 we use a result by Bestvina, Bromberg and Fujiwara described below.

Let $\mathbb{Y}$ be a set and for each $Y \in \mathbb{Y}$ let $\mathcal{C}(Y)$ be a geodesic metric space. For each $Y$ let $\pi_{Y}: \mathbb{Y} \backslash\{Y\} \rightarrow \mathcal{P}(\mathcal{C}(Y)$ ) be a function (where $\mathcal{P}(\mathcal{C}(Y))$ is the collection of all subsets of $\mathcal{C}(Y))$. Define

$$
d_{Y}^{\pi}(X, Z)=\operatorname{diam}\left\{\pi_{Y}(X) \cup \pi_{Y}(Z)\right\} .
$$

Using the enumeration in Bestvina, Bromberg and Fujiwara [6, Sections 3.1, 2.1], consider the following axioms. There exists $\xi<\infty$ so that:

(0) $\operatorname{diam}\left(\pi_{Y}(X)\right)<\xi$ for all distinct $X, Y \in \mathbb{Y}$,

(3) for all distinct $X, Y, Z \in \mathbb{Y}$ we have $\min \left\{d_{Y}^{\pi}(X, Z), d_{Z}^{\pi}(X, Y)\right\} \leq \xi$,

(4) $\left\{Y: d_{Y}^{\pi}(X, Z) \geq \xi\right\}$ is a finite set for each $X, Z \in \mathbb{Y}$.

Using the functions $d_{Y}^{\pi}$, the authors of [6] define a certain complex

$$
\mathcal{P}_{K}\left(\left\{\left(\mathcal{C}(Y), \pi_{Y}\right)\right\}_{Y \in \mathbb{Y}}\right)
$$

with vertex set $\mathbb{Y}$, which they denote as $\mathcal{P}_{K}(\mathbb{Y})$, and turns out to be a quasi-tree [6, Section 2.3].

Let $\mathcal{C}\left(\left\{\left(\mathcal{C}(Y), \pi_{Y}\right)\right\}_{Y \in \mathbb{Y}}\right)$ be the path metric space consisting of the union of all $\mathcal{C}(Y)$ 's and edges of length 1 connecting all points in $\pi_{X}(Z)$ to all points in $\pi_{Z}(X)$ whenever

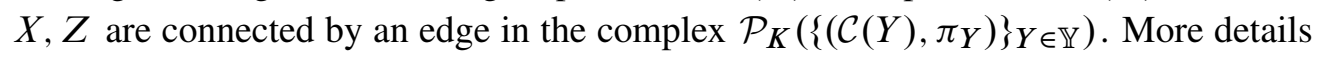
of this construction are found in [6, Section 3.1].

The only result from [6] that we need is the following.

Theorem 4.2 [6, Theorem 3.10] If $\left\{\left(\mathcal{C}(Y), \pi_{Y}\right)\right\}_{Y \in \mathbb{Y}}$ satisfies Axioms (0), (3) and (4) and each $\mathcal{C}(Y)$ is a tree then $\mathcal{C}\left(\left\{\left(\mathcal{C}(Y), \pi_{Y}\right)\right\}_{Y \in \mathbb{Y}}\right)$ is a quasi-tree (ie, it is quasiisometric to a tree). 
To apply this theorem in the context of Theorem 4.1, we fix a Cayley graph $\Gamma(G, S)$ with word metric $d$ of the relatively hyperbolic group $G$ as in Definition 2.3. Now let $\mathbb{Y}$ be the collection of all left cosets of peripheral subgroups in $G$. For each such left coset $Y=g H_{i}$ we denote by $\mathcal{C}(Y)$ the subgraph $g \Gamma\left(H_{i}, S \cap H_{i}\right)$ in $\Gamma(G, S)$, which contains $Y$ and is a copy of the Cayley graph of $H_{i}$. Let $\pi_{Y}$ be a closest point projection on $\mathcal{C}(Y)$ for each $Y \in \mathbb{Y}$. Namely, for each $x \in X$, let $\pi_{Y}(x)$ be the subset of $\mathcal{C}(Y)$ minimizing the function $d(x, \cdot)$, and let $\pi_{Y}(X)=\bigcup_{x \in X} \pi_{Y}(x)$.

As peripheral subgroups are undistorted in $G$ [24, Lemma 4.15] we can, for our purposes, identify $\mathcal{C}(Y)$ with the corresponding subset of (the Cayley graph of) $G$.

Lemma 4.3 The collection $\left\{\left(\mathcal{C}(Y), \pi_{Y}\right)\right\}_{Y \in \mathbb{Y}}$ satisfies Axioms (0), (3) and (4).

Proof For some constant $C$, the following holds [43]:

- $\operatorname{diam}\left(\pi_{Y}\left(Y^{\prime}\right)\right) \leq C$ whenever $Y \neq Y^{\prime}$, and

- if for some $x, y \in G$ we have $d\left(\pi_{Y}(x), \pi_{Y}(y)\right) \geq C$ then any geodesic from $x$ to $y$ in the Cayley graph of $G$ intersects both of the balls of radius $C$ around $\pi_{Y}(x)$ and $\pi_{Y}(y)$.

The first property clearly implies Axiom (0). The fact that the second property implies Axiom (3) can be considered folklore; see [42, Lemma 2.5]. Axiom (4) follows from the fact that the right hand side of the distance formula (2-2) is finite.

\subsection{The proof of Theorem 4.1}

Let us go back to the general setting of Theorem 4.2 for a moment, with $\left\{\mathcal{C}(Y), \pi_{Y}\right\}_{Y \in \mathbb{Y}}$ as in the statement of that theorem. Suppose that we are also given another collection of geodesic metric spaces $\left\{\mathcal{C}^{\prime}(Y)\right\}_{Y \in \mathbb{Y}}$, indexed by the same set $\mathbb{Y}$, and coarsely Lipschitz maps $f_{Y}: \mathcal{C}(Y) \rightarrow \mathcal{C}^{\prime}(Y)$, for $Y \in \mathbb{Y}$, with uniform constants. Observe that if axioms (0), (3) and (4) hold for $\left\{\left(\mathcal{C}(Y), \pi_{Y}\right)\right\}_{Y \in \mathbb{Y}}$, then they also hold for $\left\{\left(\mathcal{C}^{\prime}(Y), f_{Y} \circ \pi_{Y}\right)\right\}_{Y \in \mathbb{Y}}$.

Now suppose that each $H_{i}$ admits a quasi-isometric embedding into the product of $k$ trees. We then have coarsely Lipschitz maps $f_{i, Y}, i=1, \ldots, k$ from $\mathcal{C}(Y)$ to some tree $T_{i, Y}$. According to the observation we just made and Theorem 4.2, for each $i$ we have a map $p_{i}: G \rightarrow \mathbb{T}_{i}=\mathcal{C}\left(\left\{\left(T_{i, Y}, f_{i, Y} \circ \pi_{Y}\right)\right\}\right)_{Y \in \mathbb{Y}}$, and $\mathbb{T}_{i}$ is a quasi-tree. The last step in the proof of Theorem 4.1 is the following proposition.

Proposition 4.4 The map

$$
f=\prod p_{i} \times c: G \rightarrow \prod \mathbb{T}_{i} \times \widehat{G}
$$

is a quasi-isometric embedding, where $c: G \rightarrow \widehat{G}$ is the inclusion. 
Proof There are distance formulas for the $\mathbb{T}_{i}$ [6, Lemma 3.3, Corollary 3.12], which summed up give, for $L, \lambda, \mu$ large enough,

$$
d\left(\prod p_{i}(x), \prod p_{i}(y)\right) \approx_{\lambda, \mu} \sum_{Y \in \mathbb{Y}}\left\{\left\{d\left(\pi_{Y}(x), \pi_{Y}(y)\right)\right\}\right\}_{L} .
$$

Comparing this with the distance formula for relatively hyperbolic groups (Theorem 2.4) immediately gives the required estimates.

The map $c$ factors through Lipschitz maps $G \rightarrow X(G) \rightarrow \widehat{G}$, so the proposition implies both versions of Theorem 4.1.

\section{Three-dimensional manifold groups}

In this section we prove the following theorem.

Theorem 5.1 Let $G=\pi_{1}(M)$, where $M$ is a compact, orientable 3-manifold whose (possibly empty) boundary is a union of tori. Then eco-dim $(G)<\infty$ if and only if no manifold in the prime decomposition of $M$ has Nil geometry; in this case, $\operatorname{eco}-\operatorname{dim}(G) \leq 8$.

In any case, $\ell-\operatorname{asdim}(G) \leq 8$.

The second part of the following proposition is not needed for our purposes, but we think it is of independent interest.

Proposition 5.2 (1) If $M$ is a graph manifold with non-empty boundary then $\ell-\operatorname{asdim}\left(\pi_{1}(M)\right)=2$.

(2) If $M$ is a Haken orientable 3-manifold with non-empty boundary then we have $\operatorname{asdim}\left(\pi_{1}(M)\right) \leq 2$.

Recall that a compact orientable 3-manifold $M$ is Haken if it is irreducible and it contains a $\pi_{1}$-injective embedded surface $S$. Cutting $M$ along $S$ gives another Haken manifold, and moreover the new $\pi_{1}$-injective surface can be required to have non-empty boundary. Repeating the cutting procedure finitely many times yields a disjoint union of balls. Such sequence of cuts is called Haken hierarchy.

Proof (1) The lower bound follows from the existence of undistorted copies of $\mathbb{Z}^{2}$ in $\pi_{1}(M)$. Up to passing to a finite-sheeted cover of $M$, we can assume that $M$ fibers over $S^{1}$ by Wang and $\mathrm{Yu}$ [44], so that we have a short exact sequence

$$
1 \rightarrow F \rightarrow \pi_{1}(M) \rightarrow \mathbb{Z} \rightarrow 1,
$$


where $F$ is a free group. What is more, the content of [44, Theorem 0.7] is that a fiber intersects all Seifert components and it splits them open into products of a surface with an interval. In particular, the monodromy of the fiber bundle is a product of Dehn twists along disjoint simple closed curves (the connected components of the intersection of the fiber with the boundary of the Seifert components), and so $F$ is undistorted in $\pi_{1}(M)$. The result then follows from a direct application of [9, Theorem 0.2].

(2) We proceed by induction on the length of a Haken hierarchy for $M$ so that all surfaces involved have non-empty boundary. If $M$ is a ball, then the result trivially holds. Otherwise, $\pi_{1}(M)$ can be written as an amalgamated product $\pi_{1}\left(N_{1}\right) *_{\pi_{1}}(S) \pi_{1}\left(N_{2}\right)$ or an HNN extension $\pi_{1}\left(N_{1}\right) *_{\pi_{1}(S)}$, where $N_{i}$ is a Haken manifold admitting a strictly shorter Haken hierarchy (of the type described above) than $M$ and $S$ is a compact surface with non-empty boundary. We can then use the induction step and the fact that, when $A, B, C$ are finitely generated groups, $\operatorname{asdim}\left(A *_{C} B\right) \leq$ $\max \{\operatorname{asdim} A, \operatorname{asdim} B, \operatorname{asdim} C+1\}$ and $\operatorname{asdim}\left(A *_{C}\right) \leq \max \{\operatorname{asdim} A, \operatorname{asdim} C+1\}$ (see Dranishnikov [21] and Theorem A.1).

Remark 5.3 The second part of the proposition (in the case of toric boundary) also follows from recent results about virtual specialness of fundamental groups of 3manifolds, Liu [36], Przytycki and Wise [41], and Wise [45; 46] in view of a criterion for virtual fibering discovered by Agol [1].

We now show the following easy lemma and then prove the theorem.

Lemma 5.4 eco-dim $G_{1} * G_{2}=\max \left\{\operatorname{eco}-\operatorname{dim} G_{1}\right.$, eco-dim $\left.G_{2}\right\}$

Proof The inequality $\geq$ follows from the fact that $G_{1}, G_{2}$ are undistorted in $G=$ $G_{1} * G_{2}$. Suppose that, for $i=1,2, f_{i}: G_{i} \rightarrow \prod_{j=1}^{n} T_{j}^{i}$ are quasi-isometric embeddings. We have to show that $G$ embeds in the product of $n$ trees as well. Denote by $T$ the Bass-Serre tree of $G$. For each vertex $v$ of $T$ denote by $T_{k}^{v}$ a copy of $T_{k}^{i(v)}$, where $i(v)$ equals $i$ if the stabilizer of $v$ is conjugate to $G_{i}$. When $e$ is an edge of $T$ with endpoints $v_{1}, v_{2}$, we let $p_{e}$ be the only element of $G$ in the intersection of the left cosets of $G_{1}, G_{2}$ corresponding to $v_{1}, v_{2}$. Finally, we let $T_{k}$ be the tree obtained from $\bigcup T_{k}^{v}$ by adding an edge of length, say, 1 connecting $f_{1, k}\left(p_{e}\right)$ to $f_{2, k}\left(p_{e}\right)$ for each edge $e$ of $T$, where $f_{i, k}$ is the $k^{\text {th }}$ component of $f_{i}$ and we identify $G_{i}$ with its left coset corresponding to an endpoint of $e$.

There is a natural map $f: G \rightarrow \prod_{i=1}^{n} T_{k}$, which (up to an error bounded by 1 ) restricts to $f_{i}$ on every left coset of $G_{i}$. It is readily checked that this map is a quasi-isometric embedding (using more sophisticated technology than needed, one can use the distance 
formula and observe that, as we added edges of length 1 connecting $T_{v_{1}}$ to $T_{v_{2}}$ when $v_{1}$ is adjacent to $v_{2}$, we have $d(f(x), f(y)) \geq d_{T}(x, y)$ and $d_{T}(x, y)$ is approximately $\left.d_{\widehat{G}}(x, y)\right)$.

Proof of Theorem 5.1 Let $G_{i}$, for $i=1, \ldots, n$, be the fundamental groups of the prime factors $M_{i}$ of $M$. Then $G$ is the free product of the $G_{i}$, so that eco- $\operatorname{dim}(G)=$ $\max \left\{\operatorname{eco}-\operatorname{dim}\left(G_{i}\right)\right\}$ in view of the previous lemma, and we also have $\ell$-asdim $(G)=$ $\max \left\{\ell-\operatorname{asdim}\left(G_{i}\right)\right\}$ by [10]. In particular, we can just study the case when $M$ is prime.

We report below a list of previously known cases. Except for the last two cases, the first column indicates the geometry (of the universal cover) of $M$. The values in the table are justified below.

\begin{tabular}{ccc}
$M$ & $\ell$-asdim $\left(\pi_{1}(M)\right)$ & eco-dim $\left(\pi_{1}(M)\right)$ \\
\hline$S^{3}$ & 0 & 0 \\
$\mathbb{R}^{3}$ & 3 & 3 \\
$\mathbb{H}^{3}$, closed & 3 & 3 \\
$S^{2} \times \mathbb{R}$ & 1 & 1 \\
$\mathbb{H}^{2} \times \mathbb{R}$, closed & 3 & 3 \\
$\mathbb{H}^{2} \times \mathbb{R}$, non-closed & 2 & 2 \\
$\widehat{S L_{2} \mathbb{R}}$ & 3 & 3 \\
Nil & 3 & $\infty$ \\
Sol & 3 & 3 or 4 \\
graph manifold, closed & 3 & 3 \\
graph manifold, non-closed & 2 & 2 or 3
\end{tabular}

The bounds on $S^{3}, S^{2} \times \mathbb{R}$ and $\mathbb{R}^{3}$ are trivial. The calculation of $\ell$-asdim $\left(\mathbb{H}^{n}\right)=$ eco-dim $\left(\mathbb{H}^{n}\right)=n$ is found in Buyalo and Schroeder [16; 18]. This also gives eco-dim $\left(\mathbb{H}^{2} \times \mathbb{R}\right) \leq 3$, and $\ell$-asdim $\left(\mathbb{H}^{2} \times \mathbb{R}\right)=3$ (Dranishnikov and Smith [23, Theorem 4.3]). The spaces $\mathbb{H}^{2} \times \mathbb{R}$ and $\widetilde{S L_{2} \mathbb{R}}$ are quasi-isometric. If $M$ is not closed and has geometry $\mathbb{H}^{2} \times \mathbb{R}$ then $\pi_{1}(M)$ is virtually the product of a free group and $\mathbb{Z}$.

The discrete Heisenberg group $H$ is quasi-isometric to Nil. The result $\operatorname{asdim}(H)=$ $\ell$-asdim $(H)=3$ has been obtained by several people, for example, see Dydak and Higes [25]. On the other hand, eco- $\operatorname{dim}(H)=\infty$ as $H$ does not admit a quasi-isometric embedding into the product of finitely many metric trees, or indeed any CAT( 0$)$ space (Pauls [40]). Thus the proof in the "only if" direction is complete.

For $\ell$-asdim(Sol) $=3$ see Higes and Peng [30]. As Sol quasi-isometrically embeds in $\mathbb{H}^{2} \times \mathbb{H}^{2}$ (see, for example, de Cornulier [19, Section 9]), eco-dim(Sol) $\leq 4$. 
If $M$ is a graph manifold then $\ell$-asdim $\left(\pi_{1}(M)\right) \leq \operatorname{eco-dim}\left(\pi_{1}(M)\right) \leq 3$ by Hume and Sisto [33], and it is observed in the same paper that the equalities hold if $M$ is closed. We handled the non-closed case for $\ell$-asdim in Proposition 5.2.

There are only two cases left. First, when $M$ is a finite volume, non-closed hyperbolic manifold it is well-known that $\pi_{1}(M)$ is hyperbolic relative to virtually $\mathbb{Z}^{2}$ subgroups (Farb [26]). (Notice also that $\operatorname{asdim}\left(\pi_{1}(M)\right) \leq 3$ as $\pi_{1}(M)$ admits a coarse embedding in $\mathbb{H}^{3}$.) As $X\left(\pi_{1}(M)\right)$ is quasi-isometric to $\mathbb{H}^{3}$, by Theorem 4.1,

$$
2 \leq \ell-\operatorname{asdim}\left(\pi_{1}(M)\right) \leq \operatorname{eco}-\operatorname{dim}\left(\pi_{1}(M)\right) \leq 5 .
$$

The second case is when $M$ is non-geometric and its geometric decomposition contains at least one hyperbolic component. In this case $\pi_{1}(M)$ is hyperbolic relative to virtually $\mathbb{Z}^{2}$ and graph manifold groups, as a consequence of the Combination Theorem (Dahmani [20, Theorem 0.1]) and aforementioned fact that the hyperbolic component groups are hyperbolic relative to virtually $\mathbb{Z}^{2}$ groups (a full statement is given in Aschenbrenner, Friedl and Wilton [2, Theorem 9.2]). Therefore, by the graph manifold groups bound of [33], every peripheral group has eco-dim at most $m=3$. Note too that $\operatorname{asdim}\left(\pi_{1}(M)\right) \leq 4$ holds by Bell and Dranishnikov [4]. Thus by Theorem 1.2,

$$
\begin{aligned}
\operatorname{eco}-\operatorname{dim}\left(\pi_{1}(M)\right. & \leq \max \left\{\operatorname{asdim}\left(\pi_{1}(M)\right), m+1\right\}+m+1 \\
& =\max \{4,3+1\}+3+1=8 .
\end{aligned}
$$

Therefore, we have

$$
2 \leq \ell-\operatorname{asdim}\left(\pi_{1}(M)\right) \leq \operatorname{eco}-\operatorname{dim}\left(\pi_{1}(M)\right) \leq 8,
$$

when $M$ is non-closed, and

$$
3 \leq \ell-\operatorname{asdim}\left(\pi_{1}(M)\right) \leq \operatorname{eco}-\operatorname{dim}\left(\pi_{1}(M)\right) \leq 8,
$$

when $M$ is closed. The lower bound in the last case follows from the fact that the asymptotic dimension is greater or equal to the virtual cohomological dimension for groups of type FP (Dranishnikov [22]).

\section{Appendix A: Asymptotic dimension of HNN extensions}

The proof of the following theorem follows almost verbatim the arguments in Dranishnikov [21].

Theorem A.1 Let $A, C$ be finitely generated groups. Then

$$
\operatorname{asdim}\left(A *_{C}\right) \leq \max \{\operatorname{asdim}(A), \operatorname{asdim}(C)+1\} .
$$


We require the following notation. For $X$ a metric space, we will say that $(r, d)$ $\operatorname{dim} X \leq n$ if there exists a $d$-bounded cover of $X$ with Lebesgue number at least $r$ and 0 -multiplicity at most $n+1$. We will say that a family $\left\{X_{i}\right\}$ of metric spaces satisfy $\operatorname{asdim} X_{i} \leq n$ uniformly if for every $r>0$ there exists $d$ so that $(r, d)$-dim $X_{i} \leq n$. A partition of the metric space $X$ is a presentation of $X$ as a union of subspaces with pairwise disjoint interiors. The proof of Theorem A.1 uses the criterion below.

Theorem A.2 [21, Partition Theorem] Let $X$ be a geodesic metric space. Suppose that for every $R>0$ there exists $d>0$ and a partition $X=\bigcup_{i \in \mathbb{N}} W_{i}$ where asdim $W_{i} \leq n$ uniformly and with the property that $(R, d)-\operatorname{dim}\left(\bigcup \partial W_{i}\right) \leq n-1$ (with the restriction of the metric of $X)$. Then $\operatorname{asdim}(X) \leq n$.

We now do some preliminary work.

Fix a generating system $S_{A}$ of $A$ and let $t$ be the stable letter of the HNN extension. Let $d$ be the associated word metric on $G=A *_{C}$. The group $G$ acts on its Bass-Serre tree whose vertices are left cosets of $A$ in $G$ and whose edges are labeled by left cosets of $C$. The endpoints of the edge $g C$ are $g A$ and $g t A$. Denote by $K$ the graph dual to the Bass-Serre tree. We will denote the simplicial metric in $K$ by $|\cdot, \cdot|$, and we let $|u|=|u, C|$. Notice that for each pair of vertices in $K$ there exists a unique geodesic connecting them. Let $\pi: G \rightarrow K$ be the map $g \mapsto g C$.

Remark A.3 $\pi$ extends to a simplicial map of the Cayley graph of $G$. In particular, $\pi$ is 1 -Lipschitz.

In fact, let $s \in S_{A}$. Then for each $g \in G$ we have $g s A=g A$, so that the edges $g C$ and $g_{s} C$ of the Bass-Serre tree share the endpoint $g A$, which is exactly saying that the vertices $g C, g_{S} C$ of $K$ are connected by an edge. Similarly, the edges $g C$ and $g t C$ of the Bass-Serre tree share the endpoint $g t A$.

We divide $K$ into two parts $K_{0}, K_{1}$ intersecting only at the base vertex $C$, where $K_{1}$ contains the edges corresponding to $t A$. Let $\bar{d}$ be the graph metric on $K$, and denote by $B_{r}^{1}$ the closed $r$-ball in $K_{1}$ centered at $C$, where $r$ will always denote an integer. We will write $v \leq u$, where $u, v \in K^{(0)}$, if $v$ lies on the geodesic segment $[C, u]$ (notice that this is a partial order). For $u \in K^{(0)}$ with $u \neq C$ and $r>0$ denote

$$
K^{u}=\left\{v \in K^{(0)} \mid v \geq u\right\} \quad \text { and } \quad B_{r}^{u}=\left\{v \in K^{u}|| v|\leq| u \mid+r\right\} .
$$

Notice that if $u=g C \in K_{1}$ then $B_{r}^{u}=g B_{r}^{1}$ and $K^{u}=g K_{1}$. In particular, $\pi^{-1}\left(B_{r}^{u}\right)=$ $g \pi^{-1}\left(B_{r}^{1}\right)$ and $\pi^{-1}\left(K^{u}\right)=g \pi^{-1}\left(K_{1}\right)$.

We say that $F \subseteq G$ separates $H_{1}, H_{2} \subseteq G$ if all paths in the Cayley graph connecting $H_{1}$ to $H_{2}$ intersect $F$. Set $D_{R}=\{x \in G \mid d(x, C)=R\} \cap \pi^{-1}\left(K_{1}\right)$, for $R \in \mathbb{N}$. For $u=g C \in K_{1}$ denote $D_{R}^{u}=g D_{R}$ and notice that $\pi\left(D_{R}^{u}\right) \subseteq B_{R}^{u}$. 
Lemma A.4 Let $u \in K_{0}^{(0)}, v \in K^{(0)}$ be so that either $v<u$ or $v$ is incomparable with $u$. Then $D_{R}^{u}$ separates $\pi^{-1}(v)$ and $\pi^{-1}\left(u^{\prime}\right)$ whenever $u<u^{\prime}$ and $\left|u^{\prime}\right|-|u|>R$.

Proof We will show that $D_{R}$ separates $\pi^{-1}\left(K_{0}\right)$ and $\pi^{-1}\left(u^{\prime}\right)$ if $\left|u^{\prime}\right|>R$ and $u^{\prime} \in K_{1}$, using the action of $G$ then yields the required statement. As $\pi$ is 1 -Lipschitz we have $d\left(C, \pi^{-1}\left(u^{\prime}\right)\right)>R$ so that $D_{R}$ separates $C$ and $\pi^{-1}\left(u^{\prime}\right)$. To complete the proof notice that $C$ separates $\pi^{-1}\left(K_{0}\right)$ and $\pi^{-1}\left(K_{1}\right)$ (as their images through $\pi$ are separated by the vertex labeled $C$ ).

Lemma A.5 Suppose $R \leq r / 4$. Then $d\left(g D_{R}, g^{\prime} D_{R}\right) \geq 2 R$ whenever $g, g^{\prime} \in G$ with $g C, g^{\prime} C \in K_{1},|g C|,\left|g^{\prime} C\right| \in r \mathbb{N}$ and $g C \neq g^{\prime} C$.

Proof Set $u=g C, u^{\prime}=g^{\prime} C$. Suppose first that $|u| \neq\left|u^{\prime}\right|$. As $\pi\left(g D_{R}\right) \subseteq B_{R}^{u}$ and $\bar{d}\left(B_{R}^{u}, B_{R}^{u^{\prime}}\right) \geq r-R \geq 3 R$, and in view of the fact that $\pi$ is 1 -Lipschitz, we get $d\left(g D_{R}, g^{\prime} D_{R}\right) \geq 3 R$.

Suppose instead $|u|=\left|u^{\prime}\right|$ and pick $x \in g D_{R}, y \in g^{\prime} D_{R}$. Every path in $K$ between $\pi(x)$ and $\pi(y)$ passes through $u$ and $u^{\prime}$. This applies in particular to the projection of a geodesic $\gamma$ in the Cayley graph of $G$, so that $\gamma$ intersects $g C$ and $g^{\prime} C$. Since $d(x, g C), d\left(y, g^{\prime} C\right)=R$, we get $d(x, y) \geq 2 R$.

Lemma A.6 For $m \in \mathbb{N}$, let $(A t)^{m}=\bigcup A t^{\epsilon_{1}} \cdots A t^{\epsilon_{m}} \subseteq A *_{C}$, where each $\epsilon_{i}$ equals 0 or 1 . Then

$$
\operatorname{asdim}(A t)^{m} \leq \max \{\operatorname{asdim} A, \operatorname{asdim} C+1\}
$$

for every $m$.

Proof $(A t)^{m}$ admits a coarse embedding in $\pi_{1}(\mathcal{G})$, where $\mathcal{G}$ is a graph of groups so that all vertex groups are isomorphic to $A$, all edge groups are isomorphic to $C$ and the underlying graph is a tree. Repeated applications of [21, Theorem 2.1] give $\operatorname{asdim} \pi_{1}(\mathcal{G}) \leq \max \{\operatorname{asdim} A, \operatorname{asdim} C+1\}$, and hence the same holds for $(A t)^{m}$.

We are now ready for the proof of the theorem.

Proof of Theorem A.1 Set $n=\max \{\operatorname{ardim}(A), \operatorname{asdim}(C)+1\}$. Once we show $\operatorname{asdim}\left(\pi^{-1}\left(K_{0}\right)\right), \operatorname{asdim}\left(\pi^{-1}\left(K_{1}\right)\right) \leq n$, the conclusion follows from the Finite Union Theorem [3]. We will show the latter, using the Partition Theorem, Theorem A.2. Fix $R>0$ and take $r>4 R$. By Lemma A.4 we can write $G=X_{+} \cup X_{-}$with $X_{+} \cap X_{-}=D_{R}$ so that $X_{+} \subseteq \pi^{-1}\left(K_{1}\right)$, and $\pi^{-1}\left(K_{0}\right) \subseteq X_{-}$, and $D_{R}$ separates $X_{+} \backslash D_{R}$ and $X_{-} \backslash D_{R}$. For each $u \in K_{1}^{(0)}$ fix $g_{u}$ so that $u=g_{u} C$. Set $X_{ \pm}^{u}=g_{u}\left(X_{ \pm}\right)$, 
$V_{r}=X_{+} \cap\left(\bigcap_{|u|=r} X_{-}^{u}\right)$ and $V_{r}^{u}=g_{u}\left(V_{r}\right)$. It is readily seen that $\pi\left(V_{r}\right) \subseteq B_{r+R}^{1}$ and that

$$
\pi^{-1}\left(K_{1}\right)=\bigcup_{|u| \in r \mathbb{N}^{+}} V_{r}^{u} \cup N_{R}^{+}(C),
$$

where $N_{R}^{+}(C)=N_{R}(C) \cap \pi^{-1}\left(K_{1}\right)$. If $|u|,|w| \in r \mathbb{N}^{+}, V_{r}^{u} \cap V_{r}^{w} \neq \varnothing$ and $u \neq w$ then either $u<w$ and $|w|=|u|+r$ or, vice versa, $w<u$ and $|u|=|w|+r$. Also, if $V_{r}^{u} \cap V_{r}^{w} \neq \varnothing$ and $u<w$ then $V_{r}^{u} \cap V_{r}^{w}=D_{R}^{w}$ for $D_{R}^{w}=g_{w} D_{R}$. Putting these facts together we get:

$$
Z=\bigcup_{|u| \in r \mathbb{N}^{+}} \partial V_{r}^{u}=\bigcup_{|u| \in r \mathbb{N}^{+}} D_{R}^{u}
$$

As $D_{R}$ is coarsely equivalent to $C$, there is, for some $d>0$, an $(R, d)$-cover $\mathcal{U}$ of $D_{R}$ (ie, the Lebesgue number of $\mathcal{U}$ is at least $R$ and $\mathcal{U}$ is $d$-bounded) with 0 -multiplicity at most $n$. By Lemma A.5, $\bigcup_{|u| \in r \mathbb{N}} g_{u} \mathcal{U}$ is an $(R, d)$-cover of $Z$, and this witnesses the fact that $(R, d)-\operatorname{dim}(Z) \leq n-1$. Finally, notice that $\pi^{-1}\left(B_{s}^{1}\right) \subseteq(A t)^{s+1}$ so that $\operatorname{asdim} B_{s}^{1} \leq n$, for each $s \in \mathbb{N}$. In particular, asdim $\pi^{-1}\left(B_{r+R}^{1}\right) \leq n$ and thus $\operatorname{asdim} \pi^{-1}\left(V_{r}^{u}\right) \leq n$ uniformly. Finally, asdim $N_{R}^{+}(C) \leq n-1 \leq n$, so that the Partition Theorem applies.

\section{References}

[1] I Agol, Criteria for virtual fibering, J. Topol. 1 (2008) 269-284 MR2399130

[2] M Aschenbrenner, S Friedl, H Wilton, 3-manifold groups arXiv:1205.0202

[3] G Bell, A Dranishnikov, On asymptotic dimension of groups, Algebr. Geom. Topol. 1 (2001) 57-71 MR1808331

[4] G Bell, A Dranishnikov, On asymptotic dimension of groups acting on trees, Geom. Dedicata 103 (2004) 89-101 MR2034954

[5] G Bell, A Dranishnikov, A Hurewicz-type theorem for asymptotic dimension and applications to geometric group theory, Trans. Amer. Math. Soc. 358 (2006) 4749-4764 MR2231870

[6] M Bestvina, K Bromberg, K Fujiwara, Constructing group actions on quasi-trees and applications to mapping class groups arXiv:1006.1939

[7] B H Bowditch, Relatively hyperbolic groups, Internat. J. Algebra Comput. 22 (2012) 1250016, 66 MR2922380

[8] N Brodskiy, J Dydak, J Higes, A Mitra, Assouad-Nagata dimension via Lipschitz extensions, Israel J. Math. 171 (2009) 405-423 MR2520117

[9] N Brodskiy, J Dydak, M Levin, A Mitra, A Hurewicz theorem for the AssouadNagata dimension, J. Lond. Math. Soc. 77 (2008) 741-756 MR2418302 
[10] N Brodskiy, J Higes, Assouad-Nagata dimension of tree-graded spaces arXiv: 0910.2378

[11] M Burger, A Iozzi, N Monod, Equivariant embeddings of trees into hyperbolic spaces, Int. Math. Res. Not. 2005 (2005) 1331-1369 MR2152540

[12] S Buyalo, Asymptotic dimension of a hyperbolic space and the capacity dimension of its boundary at infinity, Algebra i Analiz 17 (2005) 70-95 MR2159584 English translation in St. Petersburg Math. J. 17 (2006) 267-283

[13] S Buyalo, Capacity dimension and embedding of hyperbolic spaces into the product of trees, Algebra i Analiz 17 (2005) 42-58 MR2173936 English translation in St. Petersburg Math. J. 17 (2006) 581-591

[14] S Buyalo, A Dranishnikov, V Schroeder, Embedding of hyperbolic groups into products of binary trees, Invent. Math. 169 (2007) 153-192 MR2308852

[15] S Buyalo, N Lebedeva, Dimensions of locally and asymptotically self-similar spaces, Algebra i Analiz 19 (2007) 60-92 MR2319510 English translation in St. Petersburg Math. J. 19 (2008) 45-65

[16] S Buyalo, V Schroeder, Embedding of hyperbolic spaces in the product of trees, Geom. Dedicata 113 (2005) 75-93 MR2171299

[17] S Buyalo, V Schroeder, Elements of asymptotic geometry, EMS Monographs in Mathematics, European Mathematical Society (EMS), Zürich (2007) MR2327160

[18] S Buyalo, V Shrëder, The hyperbolic dimension of metric spaces, Algebra i Analiz 19 (2007) 93-108 MR2319511

[19] Y de Cornulier, Dimension of asymptotic cones of Lie groups, J. Topol. 1 (2008) 342-361 MR2399134

[20] F Dahmani, Combination of convergence groups, Geom. Topol. 7 (2003) 933-963 MR2026551

[21] A Dranishnikov, On asymptotic dimension of amalgamated products and right-angled Coxeter groups, Algebr. Geom. Topol. 8 (2008) 1281-1293 MR2443244

[22] A Dranishnikov, Cohomological approach to asymptotic dimension, Geom. Dedicata 141 (2009) 59-86 MR2520063

[23] A Dranishnikov, J Smith, On asymptotic Assouad-Nagata dimension, Topology Appl. 154 (2007) 934-952 MR2294641

[24] C Druţu, M Sapir, Tree-graded spaces and asymptotic cones of groups, Topology 44 (2005) 959-1058 MR2153979

[25] J Dydak, J Higes, Asymptotic cones and Assouad-Nagata dimension, Proc. Amer. Math. Soc. 136 (2008) 2225-2233 MR2383529

[26] B Farb, Relatively hyperbolic groups, Geom. Funct. Anal. 8 (1998) 810-840 MR1650094 
[27] Ś R Gal, Asymptotic dimension and uniform embeddings, Groups Geom. Dyn. 2 (2008) 63-84 MR2367208

[28] M Gromov, Asymptotic invariants of infinite groups, from: "Geometric group theory, Vol. 2”, (G A Niblo, M A Roller, editors), London Math. Soc. Lecture Note Ser. 182, Cambridge Univ. Press (1993) 1-295 MR1253544

[29] D Groves, J F Manning, Dehn filling in relatively hyperbolic groups, Israel J. Math. 168 (2008) 317-429 MR2448064

[30] J Higes, I Peng, Assouad-Nagata dimension of connected Lie groups, Math. Z. 273 (2013) 283-302 MR3010160

[31] D Hume, Direct embeddings of relatively hyperbolic groups with optimal $\ell^{p}$ compression exponent arXiv:1111.6013

[32] D Hume, Embedding mapping class groups into finite products of trees arXiv: 1207.2132

[33] D Hume, A Sisto, Embedding universal covers of graph manifolds in products of trees, to appear in Proc. Amer. Math. Soc. arXiv: 1112.0263

[34] I Kapovich, N Benakli, Boundaries of hyperbolic groups, from: "Combinatorial and geometric group theory”, (S Cleary, R Gilman, A G Myasnikov, V Shpilrain, editors), Contemp. Math. 296, Amer. Math. Soc., Providence, RI (2002) 39-93 MR1921706

[35] U Lang, T Schlichenmaier, Nagata dimension, quasisymmetric embeddings, and Lipschitz extensions, Int. Math. Res. Not. 2005 (2005) 3625-3655 MR2200122

[36] Y Liu, Virtual cubulation of nonpositively curved graph manifolds arXiv:1110.1940

[37] J M Mackay, A Sisto, Quasi-hyperbolic planes in relatively hyperbolic groups arXiv: 1111.2499

[38] P W Nowak, On exactness and isoperimetric profiles of discrete groups, J. Funct. Anal. 243 (2007) 323-344 MR2291440

[39] D Osin, Asymptotic dimension of relatively hyperbolic groups, Int. Math. Res. Not. 2005 (2005) 2143-2161 MR2181790

[40] S D Pauls, The large scale geometry of nilpotent Lie groups, Comm. Anal. Geom. 9 (2001) 951-982 MR1883722

[41] P Przytycki, D T Wise, Mixed 3-manifolds are virtually special arXiv:1205.6742

[42] A Sisto, Contracting elements and random walks arXiv:1112.2666

[43] A Sisto, Projections and relative hyperbolicity, to appear in Enseign. Math. (2013)

[44] S Wang, F Yu, Graph manifolds with non-empty boundary are covered by surface bundles, Math. Proc. Cambridge Philos. Soc. 122 (1997) 447-455 MR1466648

[45] D Wise, The structure of groups with a quasiconvex hierarchy (2011) Available at http://www.math.mcgill.ca/wise/papers.html 
[46] D T Wise, From riches to raags: 3-manifolds, right-angled Artin groups, and cubical geometry, CBMS Regional Conference Series in Mathematics 117, Published for the Conference Board of the Mathematical Sciences, Washington, DC (2012)

Mathematical Institute, University of Oxford 24-29 St Giles', Oxford OX1 3LB, UK

john.mackay@maths.ox.ac.uk, sisto@maths.ox.ac.uk people.maths.ox.ac.uk/sisto/

Received: 24 November 2012 Revised: 16 January 2013 